\title{
Household pharmaceutical waste disposal in Selangor, Malaysia - policy, public perception, and current practices
}

\begin{abstract}
Although pharmaceuticals treat illnesses and prevent diseases in humans and animals, ironically, they are now among the emerging pollutants in the environment. As individuals continue to consume medicines, households can become a primary source of pharmaceutical pollutants. This paper explains relevant Malaysian law and policy on the issue. Using a questionnaire survey, it also assesses public perception concerning the environmental impact of pharmaceutical waste and the current methods of disposal that are practiced in Selangor. The survey used a convenience sampling and the data collected were analysed using statistical descriptive analysis. Most of the participants were aware that pharmaceutical waste can have an adverse impact on the environment and public health. Half of the participants disposed of their pharmaceutical waste in trash bins. About $2.9 \%$ of the respondents poured their household pharmaceutical waste (HPW) directly down the drain, while $8.8 \%$ poured them down the sink in the kitchen or toilet. The study also discovered that while $73.8 \%$ of the respondents felt that HPW should be separated from other household solid waste, only $25.2 \%$ returned their medicinal waste through the medicine return-back programme. The majority of the respondents $(82.5 \%)$ agreed that information concerning the proper disposal of HPW is insufficient. The study concludes that while the respondents were aware of the adverse impact of HPW, their practices to ensure proper disposal is discouraging. There is a need for effective unwanted medicines return-back programme as a more prudent disposal method of HPW to avoid any risk to the environment or human health.
\end{abstract}

Keyword: Emerging pollutants; Environmental risks; Hazardous waste; Regulations; Unwanted medicines; Waste disposal 